\title{
Revealing the spectral response of a plasmonic lens using low-energy electrons
}

\author{
Shuiyan Cao, ${ }^{1}$ Eric Le Moal, ${ }^{1, *}$ Florian Bigourdan, ${ }^{2}$ Jean-Paul Hugonin, ${ }^{2}$ Jean-Jacques Greffet, ${ }^{2}$ Aurélien Drezet, ${ }^{3}$ \\ Serge Huant, ${ }^{3}$ Gérald Dujardin, ${ }^{1}$ and Elizabeth Boer-Duchemin ${ }^{1}$ \\ ${ }^{1}$ Institut des Sciences Moléculaires d'Orsay (ISMO), CNRS, Univ Paris Sud, Université Paris-Saclay, F-91405 Orsay, France \\ ${ }^{2}$ Laboratoire Charles Fabry, Institut d'Optique, 91127 Palaiseau, France \\ ${ }^{3}$ Université Grenoble Alpes, Institut NEEL, F-38000 Grenoble, France and CNRS, Institut NEEL, F-38042 Grenoble, France
}

(Received 10 May 2017; revised manuscript received 31 August 2017; published 11 September 2017)

\begin{abstract}
Plasmonic lenses, even of simple design, may have intricate spectral behavior. The spectral response of a plasmonic lens to a local, broadband excitation has rarely been studied despite its central importance in future applications. Here we use the unique combination of scanning tunneling microscopy (STM) and angle-resolved optical spectroscopy to probe the spectral response of a plasmonic lens. Such a lens consists of a series of concentric circular slits etched in a thick gold film. Spectrally broad, circular surface plasmon polariton (SPP) waves are electrically launched from the STM tip at the plasmonic lens center, and these waves scatter at the slits into a narrow, out-of-plane, light beam. We show that the angular distribution of the emitted light results from the interplay of the size of the plasmonic lens and the spectral width of the SPP nanosource. We then propose simple design rules for optimized light beaming with the smallest possible footprint. The spectral distribution of the emitted light depends not only on the SPP nanosource, but on the local density of electromagnetic states (EM-LDOS) at the nanosource position, which in turn depends on the cavity modes of the plasmonic microstructure. The key parameters for tailoring the spectral response of the plasmonic lens are the period of the slits forming the lens, the number of slits, and the lens inner diameter.
\end{abstract}

DOI: 10.1103/PhysRevB.96.115419

\section{INTRODUCTION}

Just as light may be manipulated using glass elements, the propagation of surface plasmon polaritons (SPPs) may be controlled using a plasmonic lens [1]. Such a lens may be constructed from a series of concentric circular slits etched in a gold film. In particular, such a structure is used to transform a propagating light beam into a focused plasmon spot [2-7]. Reciprocally, a nanoscale emitter at the lens focus (e.g., a quantum dot) may transfer energy via near-field coupling to SPPs, which propagate and scatter at the circular slits into an outgoing light beam [8,9]. Thus, a plasmonic lens is both a light/SPP converter and a focusing/collimating optical component, which, like an optical antenna, links the optical near and far fields. Recently, it has been shown that the combination of such a plasmonic structure with a local, electrical source of SPPs leads to the emission of a cylindrical vector beam of light [10], a class of light beams that has various applications ranging from optical communications to manipulation of single nanoparticles [11]. The local, electrical excitation of propagating SPPs may be achieved with lowenergy electrons in a scanning tunneling microscope (STM) [12-15], or high-energy electrons in a scanning electron microscope (SEM) [16-18]. Low-energy electrical excitation of a plasmonic lens opens up new possibilities for the integration of plasmonics with electronics.

A particularity of the excitation of SPPs with electrons is that the result is broadband in frequency [15]. Notably, low-energy electrons in a tunnel junction may excite SPPs of any energy lower than the applied voltage [19]. The resulting SPP power spectrum is thus spread over the visible and infrared frequencies. This poses the question of how a plasmonic lens responds to such a broadband source, given that such

*eric.le-moal@u-psud.fr structures are usually designed for one specific energy or wavelength [20].

A clear understanding of the spectral response of a plasmonic lens in the visible and near-infrared frequency domains is pivotal for future applications. The frequency-dependent response of a plasmonic lens to a local excitation source has been addressed in a few specific cases [9,21-24], yet comprehensive studies have been missing up until now. This need is even more urgent with the recent development of novel integrated electrically driven light and SPP nanosources [25-28], which have an inherently broad power spectrum, and the rapid emergence of new fields in plasmonics that use ultrashort (and thus spectrally broad) light pulses [29,30].

In this paper, we report on the spectral response of a plasmonic lens consisting of a circular slit grating etched in a gold film. Low-energy electrons tunneling from the tip of an STM are used to electrically excite the plasmonic lens and the resulting light is analyzed using far-field optical microscopy and spectroscopy (see methods in Sec. II). From Fourier-space optical images and angle-resolved optical spectra, we obtain the spectral dependence of the emission pattern (see Secs. III A-III C). We show that the spectral response of such a plasmonic lens may be tuned by varying its inner diameter, slit period, and number. In particular, the spectral response may be tailored in such a way that the plasmonic lens yields angularly narrow light beams for a large portion of the broad energy range of SPPs excited with electrons. We demonstrate that the emission pattern of the plasmonic lens essentially results from far-field interference (see Sec. III C), whereas the spectral response is strongly affected by the dispersion relation of the slit grating (Sec. III G) and the cavity modes supported by the inner disk of the plasmonic structure (Sec. III F), as well as the dissipative losses in the metal (Sec. IIIE). Notably, the results of this study provide design rules for electrically driven, low-energy light beam microsources based 


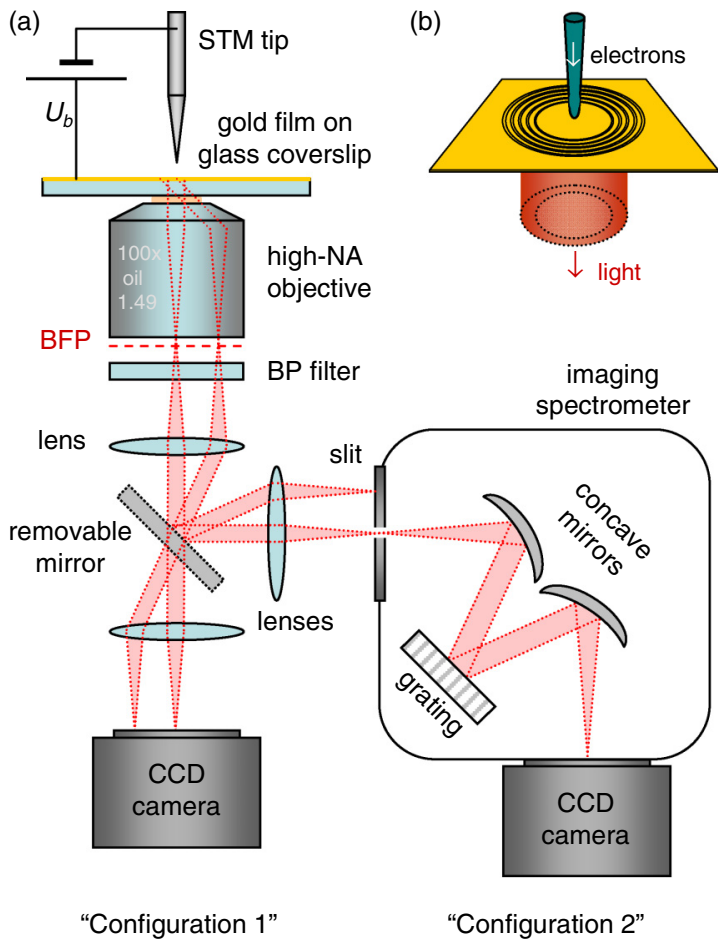

FIG. 1. (a) Experimental setup and (b) the schematic representation of the experiment. The inelastic tunnel electrons from the tungsten tip of an STM electrically excite SPPs in the center of a plasmonic lens (concentric slits etched in a gold film, typical grating period $700 \mathrm{~nm}$, typical inner diameter $5 \mu \mathrm{m}$ ). Light is collected through the substrate using a high numerical aperture, oil immersion objective lens. In (a), BFP stands for back focal plane (BP stands for bandpass and $100 \times$ refers to the magnification of the microscope objective). Again in this panel, two light beams from the sample, represented by red dotted lines, show how the Fourier-space optical image forms on the CCD camera and on the entrance slit of the spectrometer; the beam parallel with the optical axis is focused in the center of the image while the tilted beam arrives off center. One can switch from one detection pathway to another (i.e., from configuration 1 to 2 and vice versa) using a removable mirror.

on plasmonic microstructures and tunnel nanojunctions in view of integrated device applications (see Secs. III D and IV).

\section{EXPERIMENTAL METHODS}

The experimental setup and the principle of the experiment are introduced in Fig. 1. In particular, a new one-shot technique for measuring the dispersion relation is presented (configuration 2). The setup consists of a commercial STM/AFM head operating in ambient conditions and mounted on top of an inverted optical microscope [13,31]. A nanopositioning stage holds the sample between the STM tip and the objective lens. The sample is biased to $U_{b}=2.8 \mathrm{~V}$ while the tip is grounded. The tunnel current set point $I_{t}$ is in the range of 1-6 nA. STM tips are electrochemically etched tungsten wires. The light emitted in the substrate is collected using a high numerical aperture (NA $=1.45$ or 1.49 ) oil-immersion objective lens. As shown in Fig. 1(a), a removable mirror is used to switch between two configurations of the setup, where light is either (configuration 1) directly imaged on a water-cooled CCD camera or (configuration 2) spectrally dispersed by a Czerny-Turner imaging spectrometer equipped with a water-cooled CCD camera. The angular distribution of the emitted light is determined from the Fourier plane image obtained using configuration 1 of the setup. We use bandpass filters to select narrow energy ranges within the visible and near-infrared spectrum (from emission wavelengths of 550-900 $\mathrm{nm}$ ). Thus we record a series of spectrally filtered Fourier-space images [32,33]. To further resolve the spectral dependence of the angular emission pattern, we use configuration 2 of the setup, where the Fourier-space image is formed on the entrance slit of the spectrometer (this feature is new compared to the setup used in our previous publications). Thus, we record an image whose axes are the emission wavelength and the angular coordinate $n \sin \theta$, respectively, where $\theta$ is the emission angle in the substrate and $n$ is the substrate's refractive index. Such an image contains the energy-momentum dispersion relation of the excited modes, since the in-plane wave-vector component is $k_{\|}=k_{0} n \sin \theta$, where $k_{0}$ is the free-space photon wave vector. This approach is similar to other angle-resolved spectroscopy techniques that have been developed for cathodoluminescence in a scanning electron microscope (SEM) [34,35]. Alternatively, the CCD image may be vertically integrated before the readout (i.e., intensity is integrated over the angular coordinate) in order to obtain the spectral distribution of the light emitted in the substrate. In either case, the collection angle for the emitted light is limited to the angle defined by the finite aperture of the objective lens, i.e., to $\theta_{\max } \approx 79^{\circ}$ when $\mathrm{NA}=1.49$. The spectra (not the images) shown in the paper are corrected for the spectral response of the instrument. This correction includes the transmission of the microscope objective, the scattering efficiency of the grating, and the quantum efficiency of the CCD camera (see Supplemental Material [36]).

The plasmonic structure is prepared by focused ion beam (FIB) etching of a gold film that is $200 \mathrm{~nm}$ thick, and prepared by thermal evaporation on a glass coverslip coated with a $100 \mathrm{~nm}$-thick layer of indium tin oxide (ITO). The design of the structure is either a circular grating of five concentric slits or a single circular slit. The slit width is $300 \mathrm{~nm}$ and the etch depth is $200 \mathrm{~nm}$. Structures with central disks of inner diameters $D=3,4,5$, and $6 \mu \mathrm{m}$ are examined. Grating periods $P=$ 600,700 , and $800 \mathrm{~nm}$ are considered.

In all the experiments discussed below, the STM tip is located in the center of the plasmonic lens. Inelastic electron tunneling (IET) from the tip to the sample leads to the excitation of a two-dimensional (2D)circular surface plasmon wave that propagates isotropically away from the tip location. This results from the fact that the IET excitation may be considered as a pointlike, out-of-plane electric dipole located inside the tip-sample gap $[12,37,38]$. Here, the gold film is thick enough to prevent SPP leakage radiation in the glass; thus, only the light from SPPs scattered at the slits of the structure is detected below the substrate.

\section{RESULTS AND DISCUSSION}

\section{A. Full-spectrum angular emission patterns}

When the STM tip excitation is in the center of the plasmonic lens, the scattering of the excited SPPs at the 

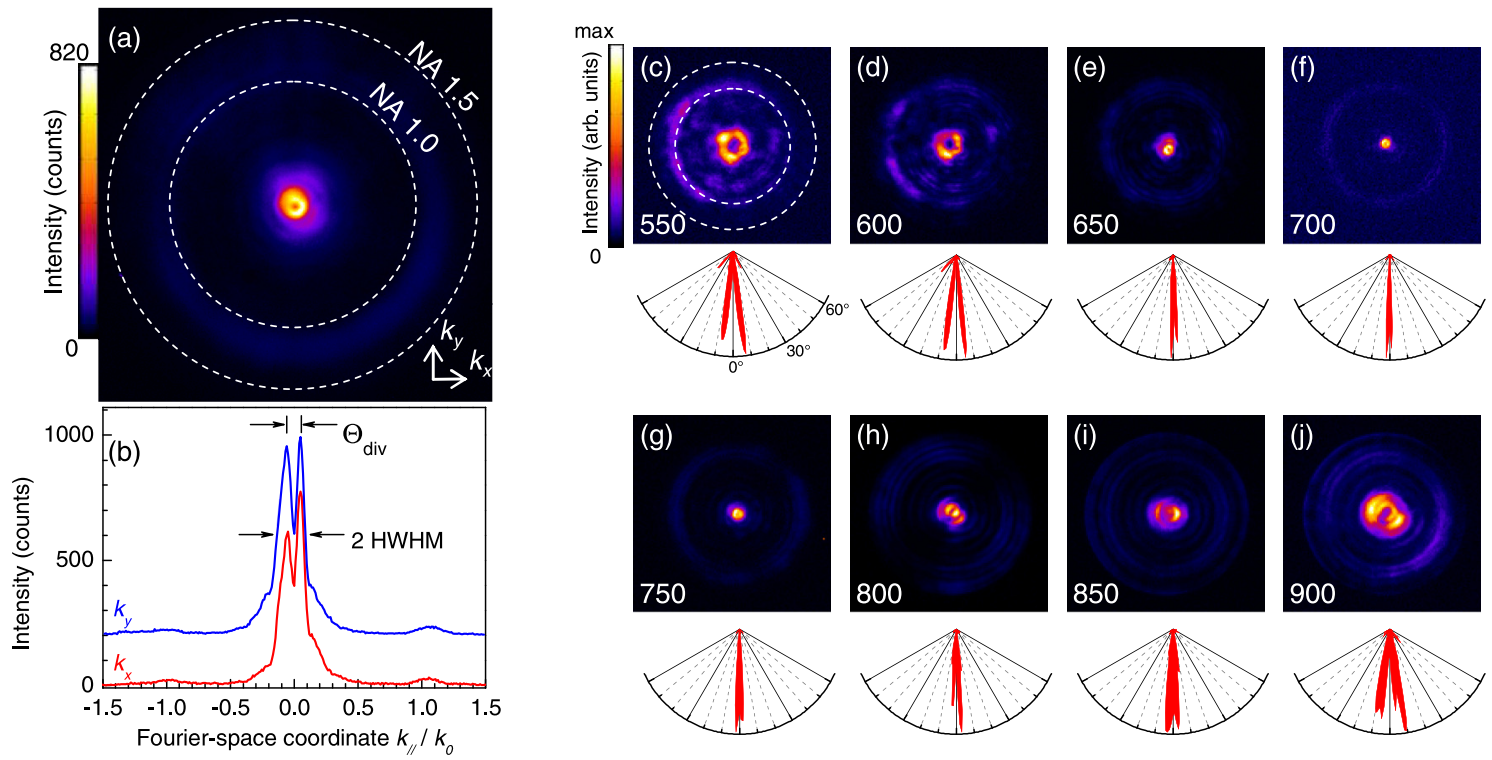

FIG. 2. Imaging the angular emission pattern at different energies for an STM-excited plasmonic lens. (a) Fourier-space optical image, in false color, revealing the angular emission pattern in the substrate. The plasmonic lens is a five-slit circular grating of period $700 \mathrm{~nm}$ and inner diameter $5 \mu \mathrm{m}$. For this image, light is collected using an objective lens of NA $=1.49$, the full emission spectrum is used (no spectral filtering) and the image resolution is $0.0098 k_{0}$ per pixel (i.e., the NA $=1.5$ circle has a diameter of 306 pixels). The STM bias is $U_{b}=2.8 \mathrm{~V}$, the current setpoint is $I_{t}=6 \mathrm{nA}$ and the acquisition time is $600 \mathrm{~s}$. (b) Intensity profiles taken from the Fourier image in (a), along the direction of the Fourier-space vectors $\mathbf{k}_{\mathbf{x}}$ and $\mathbf{k}_{\mathbf{y}}$. The profile along $\mathbf{k}_{\mathbf{y}}$ is vertically offset for clarity. The Fourier-space coordinates $\left(k_{\|}\right.$stands for $k_{x}$ or $k_{y}$ ) are normalized by the wave vector corresponding to the spectral peak wavelength in free space $k_{0}$. The angular beam divergence is defined as the difference in angle corresponding to the two peaks observed in the intensity cross sections in Fourier space. (c)-(j) Spectrally filtered Fourier-space optical images, in false color, of the light emitted in the substrate. The collected light is filtered using bandpass filters that have the following central wavelengths/bandwidths: (c) $550 / 88 \mathrm{~nm}$, (d) $600 / 14 \mathrm{~nm}$, (e) $650 / 13 \mathrm{~nm}$, (f) $700 / 13 \mathrm{~nm}$, (g) $750 / 40 \mathrm{~nm}$, (h) $800 / 40 \mathrm{~nm}$, (i) $850 / 40 \mathrm{~nm}$, and (j) $900 / 40 \mathrm{~nm}$. Intensity profiles taken from the Fourier images are shown below each image in a polar plot. For these images, light is collected using an objective lens of $\mathrm{NA}=1.45$ and the image resolution is $0.027 k_{0}$ per pixel (i.e., the NA $=1.5$ circle has a diameter of $\approx 108$ pixels) and the acquisition time is $300 \mathrm{~s}$.

slits gives rise to the emission of a cylindrical vector beam of light with radial polarization [10]. Figure 2(a) shows the resulting Fourier-space optical image for a five-slit circular grating of period $700 \mathrm{~nm}$ and inner diameter $5 \mu \mathrm{m}$. The image shows a small, doughnut-shaped spot in the center, which is characteristic of a highly collimated, radially polarized light beam emitting orthogonally to the plane of the plasmonic lens. From the intensity profiles shown in Fig. 2(b), we evaluate the angular beam divergence at $\pm 4.1^{\circ}$ with respect to the optical axis [see how beam divergence is defined in Fig. 2(b)]. This very low angular divergence as well as beaming of light along the optical axis is obtained because the scattered light is emitted in phase from the five slits of the plasmonic lens [10]. This occurs when the period of the circular grating (i.e., the radial distance between two nearest slits) equals an integral number of SPP wavelengths.

The observation of such beam collimation might seem surprising since electrical SPP excitation by IET is known to inherently be broadband [38]; thus, the phase condition mentioned above is only met for a narrow part of the emission spectrum while a large portion of spectrum of the collected light is used to obtain the Fourier image shown in Fig. 2(a). The system formed by a tungsten tip and a gold film with a junction bias of $U_{b}=2.8 \mathrm{~V}$ is known to produce an emission maximum with a photon wavelength around $\lambda_{0}=720 \mathrm{~nm}(\approx 1.7 \mathrm{eV}$ in energy) and a full-width-at-half-maximum of $\Delta \lambda_{0} \approx 200 \mathrm{~nm}$ $(\approx 0.5 \mathrm{eV})$ [13]. Nevertheless, we demonstrate below that the spectral response of such a plasmonic lens upon local, central excitation is advantageously broad, thus preserving this beaming effect for a large portion of the power spectrum of the electrical SPP source. This study, which investigates the spectral response of a plasmonic lens for an energy range greater than $1 \mathrm{eV}$ (i.e., from $\lambda_{0} \approx 550-1000 \mathrm{~nm}$ ), is possible thanks to the combination of a spatially localized, spectrally broad, accurately positionable source of SPPs (i.e., the tunnel junction under the STM tip) with the necessary optical tools to angularly and spectrally resolve the emitted light.

\section{B. Frequency dependence: a simple model}

In Figs. 2(c)-2(j), Fourier-space images obtained with different interferometric bandpass filters in front of the CCD camera are shown. The plasmonic lens is the same as the one used in Fig. 2(a) (i.e., $P=700 \mathrm{~nm}, D=5 \mu \mathrm{m}$, five slits) and the STM tip is again located in the center of the structure. The filters used have central wavelengths ranging from 550-900 nm as indicated in the bottom left corner of the images. All the images of this series exhibit a doughnut-shaped spot centered on the Fourier space origin; yet the size of this spot varies as a function of the detection wavelength. The spot is smallest for a detection wavelength of $700 \mathrm{~nm}$. As well, the polar plots below the images show that the beam 

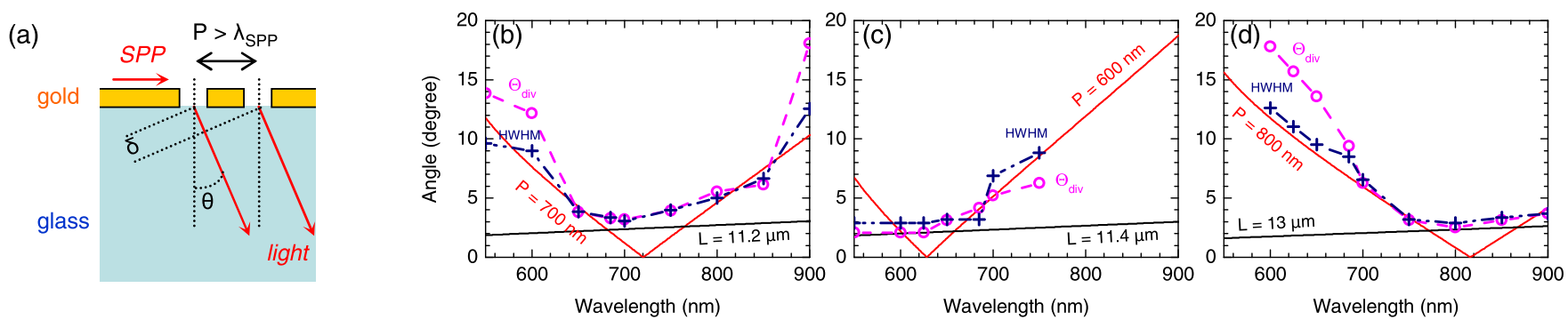

FIG. 3. Spectral variation of the angular beam divergence: a simple model and the experimental results. (a) Sketch of the scattering of SPPs into light at two slits etched in a gold film on glass. The SPPs propagate from the left-hand side of the figure. The light emitted in the substrate from the two slits interferes constructively in the direction denoted by the angle $\theta$. $\delta$ is the optical path difference for the light emitted at $\theta$ at the two slits. If the period $P$ of the slit grating is longer than the SPP wavelength $\lambda_{\mathrm{SPP}}$, then the SPPs scattered into light interfere constructively in the forward direction. For $P<\lambda_{\mathrm{SPP}}$, constructive interference is in the backward direction. Only first-order diffraction is considered here. [(b) to (d)] The experimental values of the angular beam divergence [see Fig. 2(b) for definitions] measured for three different five-slit circular gratings are compared with the angle of constructive interference $\theta$ (red line) calculated using the model shown in (a). The grating period is $P=700 \mathrm{~nm}$ in (b), $P=600 \mathrm{~nm}$ in (c) and $P=800 \mathrm{~nm}$ in (d). The inner diameter of the plasmonic lens is $D=5 \mu \mathrm{m}$ in (b) and $D=6 \mu \mathrm{m}$ in (c) and (d). The theoretical lower bound for the angular beam divergence due to the finite size of the slit grating is shown by the black line. $L$ is the outer diameter of the plasmonic lens. The experimental data are obtained from the analysis of spectrally filtered Fourier-space images following the method described in Fig. 2.

divergence is the largest at the extrema of the investigated spectral range. To confirm these observations and using the definition of beam divergence given in Fig. 2(b), we determine the angular divergence of the light beam from the Fourier-space optical images, and plot it versus the emission wavelength as shown in Figs. 3(b)-3(d). The angular beam divergence minimum is found to be $3.2^{\circ}$ at $\lambda_{0}=700 \mathrm{~nm}$. For the energy corresponding to a photon wavelength of $\lambda_{0}=700 \mathrm{~nm}$ in vacuum, the SPP wavelength on an ideal smooth gold/air interface is $\lambda_{\mathrm{SPP}} \approx 678 \mathrm{~nm}$, which is indeed close to the period of the circular slit grating. Interestingly, the angular beam divergence remains lower than $4.0^{\circ}$ within a $100-\mathrm{nm}$ wide wavelength range around $700 \mathrm{~nm}$, and lower than $7.0^{\circ}$ until $\lambda_{0}=850 \mathrm{~nm}$. In order to explain this effect, we use a simple two-slit interference model (see details below) to determine the spectral dependence of the angular beam divergence.

Figure 3(a) illustrates the principle of the simple 2D scalar model used. We consider only two adjacent slits, which scatter the incoming SPPs into light in the substrate, and model these slits as two coherent point sources, separated by the slit grating period $P$. The emission from these two point sources is assumed isotropic in the glass. Thus, the light in the far field is expected to be the most intense at an angle where constructive interference occurs between the waves emitted from the two slits. Consequently, a maximum in the emission of light in the direction orthogonal to the interface only occurs if the two sources are in phase, i.e., $P=\lambda_{\text {SPP }}$. Otherwise, SPPs will preferentially scatter in the direction where the optical path difference in glass $\delta$ compensates the phase shift between the two sources. Within this simple representation, we assume that the energy-dependent SPP wavelength is that of SPPs propagating on the interface between infinite gold and vacuum half-spaces. This reads

$$
n_{\mathrm{SPP}}^{\prime} P-n_{g} \delta=\lambda_{0},
$$

where $n_{g}=1.51$ is the refractive index of glass, $\lambda_{0}$ is the photon wavelength in vacuum, and $n_{\mathrm{SPP}}^{\prime}$ is the effective index of the SPPs propagating at the gold-air interface. $n_{\mathrm{SPP}}^{\prime}$ is defined as the real part of the wave-number ratio between SPPs and photons in vacuum, as follows:

$$
n_{\mathrm{SPP}}^{\prime}=\mathfrak{R e}\left(\frac{k_{\mathrm{SPP}}}{k_{0}}\right) \cong\left(\frac{\epsilon_{m}^{\prime}}{\epsilon_{m}^{\prime}+1}\right)^{\frac{1}{2}}
$$

for $\left|\epsilon_{m}^{\prime \prime}\right| \ll\left|\epsilon_{m}^{\prime}\right|$ where $\epsilon_{m}^{\prime}$ and $\epsilon_{m}^{\prime \prime}$ are the real and imaginary parts of the frequency-dependent relative permittivity of the metal respectively. Equivalently, $n_{\mathrm{SPP}}^{\prime}=\lambda_{0} / \lambda_{\mathrm{SPP}}$. From Eq. (1), we see that constructive interference occurs for

$$
\theta=\arcsin \left(\frac{n_{\mathrm{SPP}}^{\prime}-\lambda_{0} / P}{n_{g}}\right) .
$$

Only first-order diffraction is considered here. Consequently, SPPs interfere constructively in the forward direction if $P>$ $\lambda_{\text {SPP }}$, and in the backward direction if $P<\lambda_{\text {SPP }}$. We come back now to a 3D problem where SPPs scatter on circular slits and, as a first step, we ignore the effects of the finite size of the slit grating. Thus, we find that the emitted beam has the shape of a cylinder in the far field only if $P=\lambda_{\mathrm{SPP}}$. If $P \neq \lambda_{\mathrm{SPP}}$, the emitted beam has the shape of a cone in the far field, with a beam divergence angle $\theta$ given by Eq. (3). Figures 3(b)-3(d) show the results of this simple model (see red line) along with the experimental data for three different slit grating periods: $P=600,700$, and $800 \mathrm{~nm}$. As a second step, we introduce in the model the effect of the finite size of the slit grating by including a lower bound to the angular beam divergence. Within the far-field approximation (Fraunhofer diffraction), the radiation pattern from the source has an angular spread $\Delta k$ limited by the source size $L$ through the relation $L \Delta k \gtrsim 2 \pi$. Here, light is emitted from the slits etched in the gold film; thus the source size $L$ is the outer diameter of the $N$-slit circular grating, i.e., $L=D+2(N-1) P+2 W$, where $W$ is the slit width. Light is mainly emitted orthogonally to the gold film, i.e., along the optical axis of the microscope; therefore, $\Delta k$ is equivalent to the width of the doughnut-shaped spot observed in the Fourier-space images. The above relation thus leads to a lower bound for the angular divergence of the light beam from 
(a)

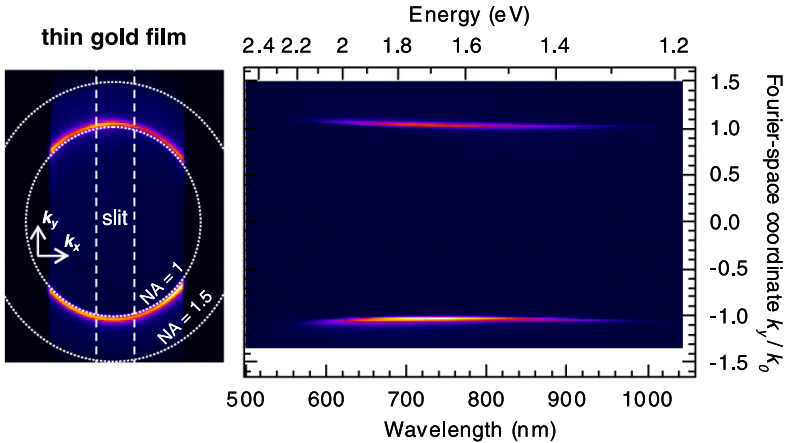

(b)

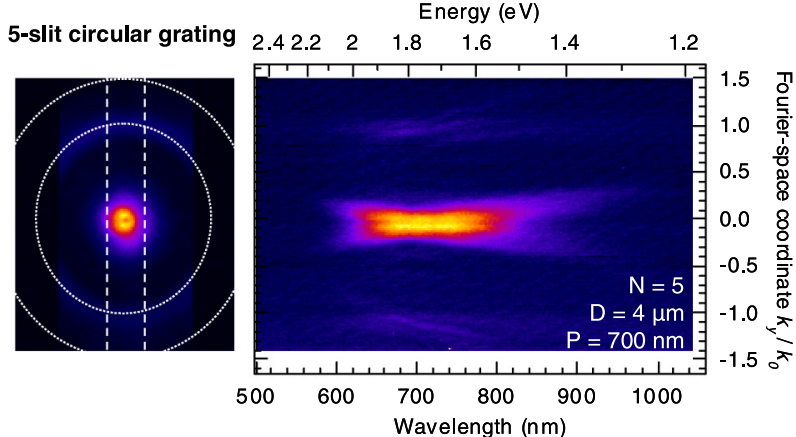

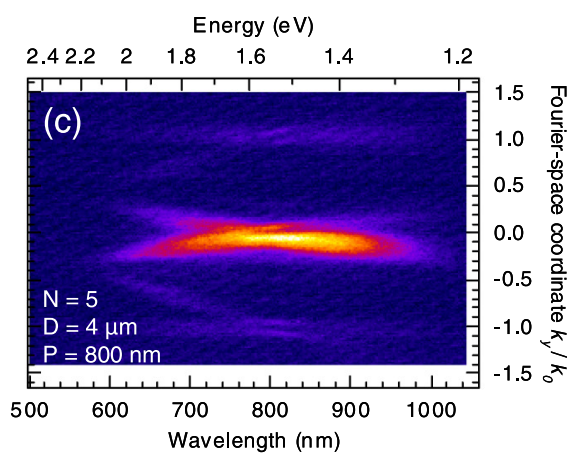
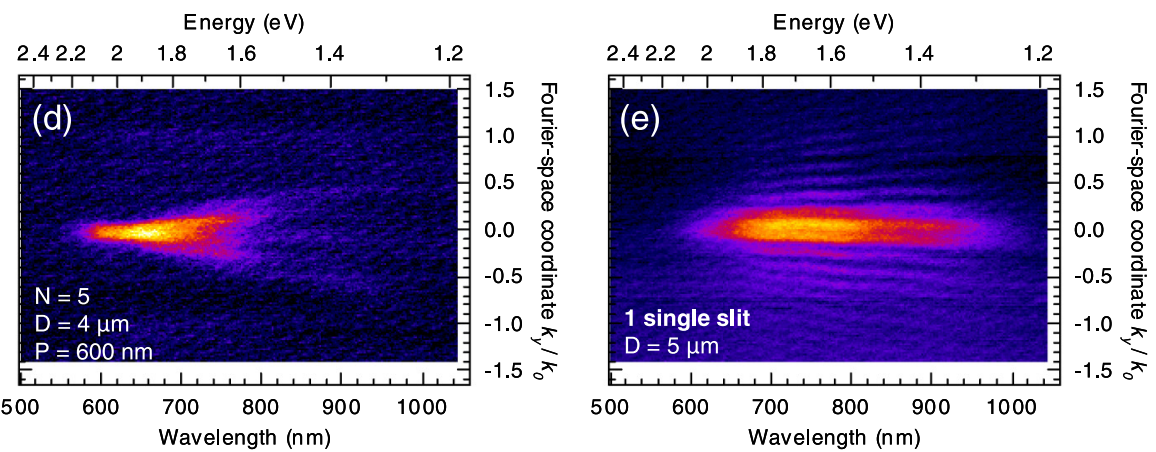

FIG. 4. Angle-resolved spectroscopy: method and results. Angular and spectral distributions of the light emitted upon STM excitation of (a) an unstructured 50-nm gold film on glass, (b)-(d) a five-slit plasmonic lens of inner diameter $D=4 \mu \mathrm{m}$ and period (b) $P=700 \mathrm{~nm}$, (c) $P=800 \mathrm{~nm}$, and (d) $P=600 \mathrm{~nm}$, and (e) a single circular slit of inner diameter $D=5 \mu \mathrm{m}$, etched in a 200-nm gold film on glass. These experimental data are measured by projecting the Fourier-space image of the emitted light on the partly closed entrance slit of the spectrometer [see Fig. 1(a), configuration 2]. The STM bias is $U_{b}=2.8 \mathrm{~V}$, the current set point $I_{t}=3$ to $4 \mathrm{nA}$ and the acquisition time is $300 \mathrm{~s}$. On the left of (a) and (b) are the Fourier-space images that are obtained when the spectrometer entrance slit is at its largest width and the image is acquired using the zeroth order of the diffraction grating (acquisition time: $100 \mathrm{~s}$ ). Dotted lines indicate the selected area when the entrance slit is partially closed for spectral measurements. The partially closed entrance slit gives rise to a spectral resolution of about $8 \mathrm{~nm}$ (i.e., $\approx 0.02 \mathrm{eV}$ at $\lambda_{0}=700 \mathrm{~nm}$ ). Along the vertical axis $\left(k_{y} / k_{0}\right)$, the resolution is limited by the CCD pixel size to 0.018 per pixel.

the plasmonic lens:

$$
\theta \gtrsim \arcsin \left(\frac{\lambda_{0}}{n_{g} L}\right) .
$$

The simple model based on Eqs. (3) and (4) reproduces the main trends of the experimentally determined spectral dependence of the angular beam divergence sufficiently. Thus the desired optimum operating frequency range and size of the plasmonic lens as well as the required angular spread may be simply obtained by choosing specific geometric design parameters (inner diameter, grating period, number of slits). Such simple control over the plasmonic lens characteristics is crucial for its future integration with a broadband excitation nanosource. Neither the model nor the spectrally filtered Fourier-space images (which are not intensity-normalized with respect to each other) provide the power spectrum of the emitted light versus its angular distribution, however. To obtain this key information, angle-resolved optical spectroscopy is necessary where the angular and spectral dependence are revealed in one single image.

\section{Angle-resolved emission spectra}

Figure 4 shows the angularly-resolved spectroscopy results obtained using configuration 2 of the experimental setup shown in Fig. 1(a). By projecting the Fourier-space image obtained from the emitted light on the entrance slit of the spectrometer, we spectrally resolve the collected light along a line in the Fourier plane. The relative position of the projected image is adjusted so that the light entering the entrance slit of the spectrometer is from a line along $\mathbf{k}_{\mathbf{y}}$ and crosses the Fourier space origin. Thus, we record an image (on the CCD camera of the spectrometer) with spectral and angular coordinates along the horizontal and vertical axes, respectively. When the system exhibits axial symmetry with respect to the optical axis, the angular dependence of the emission pattern is only radial (not azimuthal); therefore, all information about the energy-momentum dispersion (within the investigated energy range) is contained in the images shown in Fig. 4.

In Fig. 4(a), we use a thin 50-nm gold film on glass to calibrate the vertical axis $\left(k_{y} / k_{0}\right)$ of the Fourier-space images. The ring from SPP leakage radiation (see image on the left) occurs at an angle that is theoretically known from the SPP dispersion relation $\left(k_{\mathrm{SPP}} / k_{0} \approx 1.037\right.$ for $\lambda_{0}=$ $700 \mathrm{~nm}$ ). In Figs. 4(b)-4(e), we analyze the light emitted from three different plasmonic structures under the same excitation conditions as in Fig. 2. These structures are five-slit circular gratings, with an inner diameter of $D=4 \mu \mathrm{m}$ but with different grating periods $(P=600,700$, and $800 \mathrm{~nm})$, and a single circular slit with $D=5 \mu \mathrm{m}$ etched in a thick $200-\mathrm{nm}$ gold film on glass. 
The general aspect of the energy-momentum dispersion diagram for all four structures may be easily understood by drawing an analogy to Young's double-slit experiment. In the case of a single circular slit, we may assume that the light detected in one direction $k_{\|} / k_{0}$ in Fourier space results from the interference of the light emitted from two opposite point sources on the slit. Thus, a periodic fringe pattern is expected in Fourier space, with a period varying as the emission wavelength over the slit diameter [39]. This is indeed what is observed in Fig. 4(e). In a five-slit circular grating, five fringe patterns of different periods superimpose in Fourier space and coherently interfere with each other. Constructive interference in the emission direction orthogonal to the grating only occurs if the slit period is a multiple of the emission wavelength. This explains why the shape of the energy-momentum dispersion diagrams seen in Figs. 4(b)-4(d) varies with wavelength and why these bow ties shift in energy as the slit grating period is changed. The angular divergence is lowest and the light emitted in the substrate is most intense at the center of the bow tie. Around this energy value, the angular divergence and the radiated power remain almost constant (and at their optimal values) within $\mathrm{a} \approx 0.3 \mathrm{eV}$-wide range.

\section{Optimization rules for plasmonic lens design}

We can now explain why the angular beam divergence obtained from the full-spectrum Fourier-space image shown in Fig. 2(a) is very close to its lowest value despite the spectral broadness of the electrical SPP nanosource. Two main effects determine the angular beam divergence of our electrical light microsource: the spectral width of the SPP nanosource and the finite size of the structure at which SPPs scatter into light. When the latter dominates over the former, the angular beam divergence remains almost constant over the emission spectrum. Thus the size of the plasmonic microstructure should be decreased to the smallest possible diameter where the angular width of the emitted beam is not limited by the size effect. Beyond this point, further downsizing of the plasmonic microstructure is inefficient, since it broadens the angular distribution of the emitted light without improving the matching between the spectral response of the plasmonic structure and the power spectrum of the SPP nanosource. Obtaining optimal light beaming while keeping the smallest possible spatial footprint thus requires that the spectral width and size effects contribute equally to the angular beam divergence of the source. From Eqs. (3) and (4) for small $\theta \approx 0$, we evaluate the contributions of these two effects at $\Delta \lambda_{0} /\left(n_{g} P\right)$ and $\lambda_{0} /\left(n_{g} L\right)$, respectively, where $\Delta \lambda_{0}$ and $\lambda_{0}$ are the spectral width and central wavelength of the source $(P$ and $L$ are the period and outer diameter of the slit grating and $n_{g}$ is the substrate's refractive index). The best tradeoff between narrow light beaming and small spatial footprint is thus obtained when using a plasmonic lens with period-to-size ratio $P / L \approx \Delta \lambda_{0} / \lambda_{0}$.

The data shown in Figs. 4(b) and 4(e) also reveal the advantages and drawbacks of increasing the number of slits $N$ of the structure. From Fourier-space images, we evaluate the angular divergence of the light beam emitted from the five-slit circular grating $(D=4 \mu \mathrm{m}$ and $P=700 \mathrm{~nm})$ and the single circular slit $(D=5 \mu \mathrm{m})$ at $4.4^{\circ}$ and $5.6^{\circ}$, respectively (data not shown). Therefore, the latter structure, which is almost half the size of the former $(5.6 \mu \mathrm{m}$ in outer diameter, as compared to $10.2 \mu \mathrm{m}$ ), has an angular beam divergence that is only $\approx 25 \%$ higher. Moreover, using one circular slit rather than five extends the energy range for which optimal beaming and power radiation are obtained. However, the emission pattern obtained from a single circular slit exhibits multiple secondary lobes of non-negligible intensity, which persist within all the investigated energy range [see Fig. 4(e)]. This off-axis emission represents an important power loss for a light beam source. Furthermore, in an applied context, cross-talk issues may occur when several of these sources are used to transfer information to distinct receptors. In this regard, a multiple-slit structure is advantageous.

\section{E. Material effects}

A feature that all panels of Fig. 4 have in common is the strong damping of the radiated power at an energy higher than about $2.1 \mathrm{eV}$ (i.e., $\lambda_{0} \lesssim 590 \mathrm{~nm}$ ). This is due to losses in tungsten and gold, i.e., the metals of which the STM tip and the plasmonic lens are made. Generally, the power spectrum of the emission radiated from a tunnel junction is modeled as the product of a source term given by inelastic tunneling statistics $\left(\propto\left(e U_{b}-\hbar \omega\right)\right.$, for $\hbar \omega \leqslant e U_{b} ; 0$ otherwise [19]) and an emission term related to the local density of electromagnetic states (EM-LDOS) [40,41]. As a result, the radiation has a broad power spectrum that spreads over the infrared and part of the visible range and falls to zero at energy $e U_{b}$ (quantum cutoff). Still, the power spectrum radiated by a W-Au tipsurface junction is known to rapidly fall beyond $2.1 \mathrm{eV}$ in energy [38], even when the tunnel junction is biased at $U_{b}=$ $2.8 \mathrm{~V}$. This is notably ascribed to interband transitions in gold. In the system that we study here, the dissipative losses in gold play a double role, since they decrease the power emitted by the SPP nanosource and they shorten the SPP propagation length, thus yielding higher propagation losses before SPP scattering from the slits into light in the far field.

\section{F. Spectral response: Effect of the plasmonic modes}

Figure 5 shows optical spectra measured for different plasmonic lenses using configuration 2 of the experimental setup with integration over the $\mathrm{CCD}$ lines before readout (i.e., the signal is integrated over emission angles). Thus, we focus on the total emitted power, which is related to the EM-LDOS at the SPP nanosource position. In this way, finer spectral features, whose energy position clearly depends on the plasmonic lens parameters $N, D$, and $P$, are made visible ( $N$ is the number of slits, $D$ is the inner disk diameter, and $P$ is the grating period). The spectrum measured on an unstructured thin $(50 \mathrm{~nm})$ gold film under the same conditions, shown in Fig. 5(b), exhibits no such features; it resembles a simple Gaussian curve. This confirms that the features seen in the plasmonic lens spectra are due to the structures etched in the gold film and not to the SPP nanosource.

The spectrum obtained from a single circular slit, shown in black in Fig. 5(c), differs significantly from that of the unstructured thin gold film; it exhibits peaks and dips. When varying $D$, the inner diameter, (data shown in the 

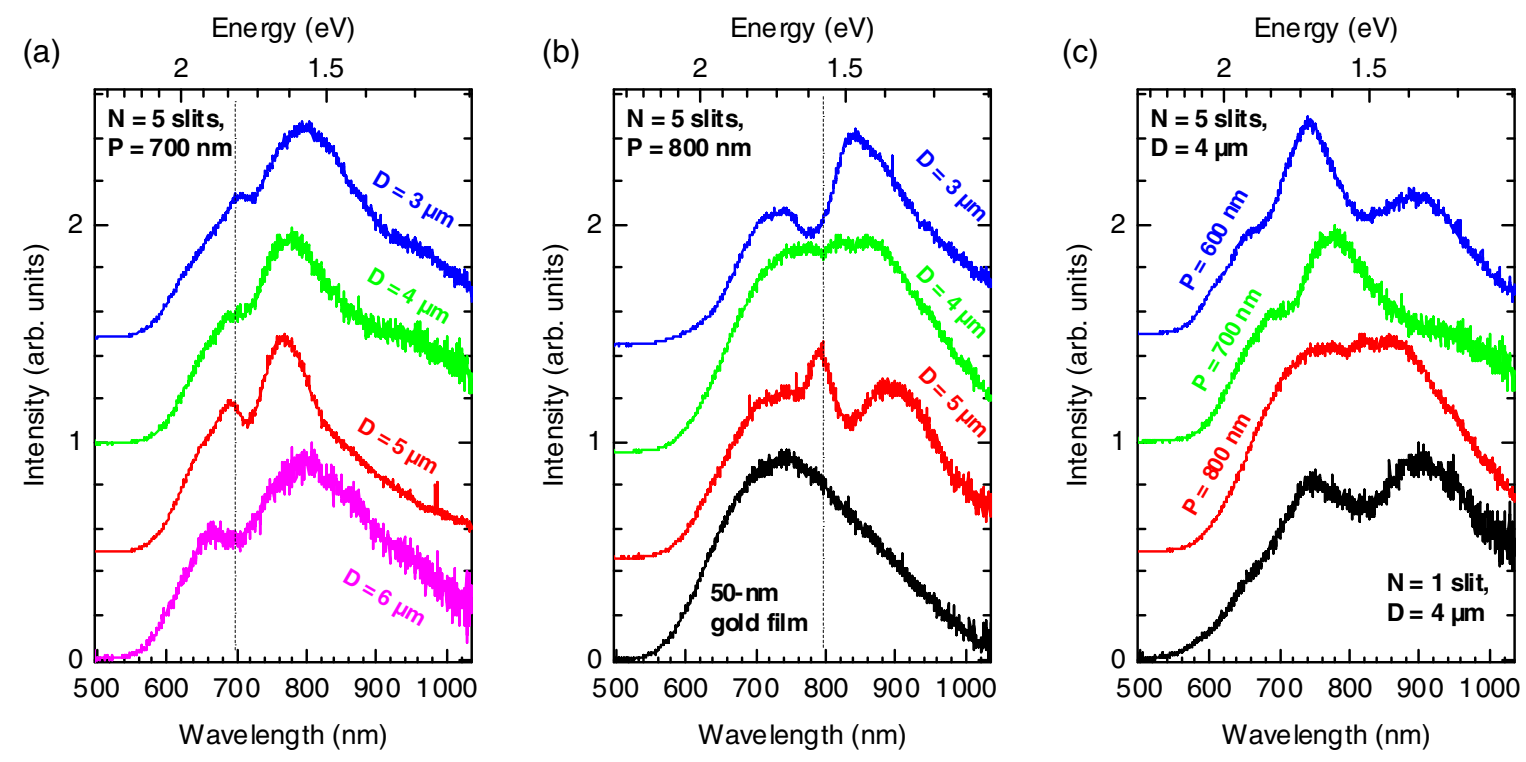

FIG. 5. Radiated power spectra: effect of the plasmonic lens parameters. Spectral distribution of the light emitted upon STM excitation of a five-slit $(N=5)$ circular grating of different inner diameters $D$ and periods $P$, etched in a 200-nm gold film on glass. The spectral dependence on $D$ with (a) $P=700 \mathrm{~nm}$ and (b) $P=800 \mathrm{~nm}$ is examined (a vertical dotted line indicates $\lambda_{0}=P$ to guide the eye). In (c), the effect of varying $P$ while keeping $D$ fixed is studied. For comparison purposes, the spectra obtained for an unstructured 50-nm gold film and for a single $(N=1)$ circular slit etched in a 200-nm gold film are also plotted in (b) and (c) (black lines), respectively. All spectra are corrected for the spectral response of the instrument, which includes the transmission of the microscope objective, the scattering efficiency of the diffraction grating and the quantum efficiency of the CCD camera used in the spectrometer. Spectra are vertically offset for clarity. The STM bias is $U_{b}=2.8 \mathrm{~V}$, the current setpoint is $I_{t}=3$ to $4 \mathrm{nA}$ and the acquisition time is $300 \mathrm{~s}$.

Supplemental Material [36]), these features shift in energy in such a way that they may be related to the cavity modes of the plasmonic structure. These modes correspond to the SPP standing waves, which form on the inner gold disk due to SPP reflection at the circular slit; the existence of such modes has been confirmed by cathodoluminescence measurements on similar structures [21].

Adding four slits and varying the slit period $P$ significantly changes the spectral response of the plasmonic structure, even though the inner diameter $D$ is kept constant [see Fig. 5(c)]. As well, if $P$ remains the same and $D$ is modified [see Figs. 5(a) and 5(b)], the peak and dip positions, relative height, and width vary. Since here the detected light is integrated over all emission angles, the spectral dependence of the emitted light that is observed as a function of slit grating period and diameter cannot be due to the phenomenon seen in Fig. 4. Instead, it is attributed to the effect of the lens parameters on the EM-LDOS. Namely, the power of the light beam emitted from the plasmonic lens must be sensitive to the EM-LDOS at the position of the SPP nanosource. Below, we explain why strong variations occur in the EM-LDOS when the emission wavelength is close to the slit grating period.

\section{G. Spectral response: Role of the slit grating}

The role of the circular-slit grating in the spectral response of the plasmonic lens is twofold. The grating, which surrounds the central disk, reflects part of the outgoing SPP wave back to the center of the disk, and also scatters part of the SPP wave into light in the out-of-plane direction. Thus, the slit grating and the central-disk cavity are coupled through the frequency- dependent Fresnel coefficients of the grating. The intensity of the light emitted from the slits depends on both the scattering coefficient of the grating and the intensity of the incident SPPs; the latter, however, depends on the SPP-mode density in the central-disk cavity, which in turn is determined by the complex amplitude reflection coefficient $r$ of the slit grating. Depending on frequency, this grating-cavity coupling may have a strong enhancing or quenching effect on the spectral response of the plasmonic lens and the magnitude of this effect depends on $r$.

We have numerically calculated the complex amplitude SPP reflection coefficient $r$ and transmission coefficient $t$ of a single linear slit grating using a 2D model for normal SPP wave incidence $[42,43]$. The squared moduli $R=|r|^{2}$ and $T=|t|^{2}$ are the intensity Fresnel reflection and transmission coefficients and satisfy the relationship $R+T+S+A=1$ with $S$ the out-of-plane SPP-to-light scattering coefficient and $A$ the dissipative loss (absorption) coefficient. In Fig. 6, $R$ and $T$ are plotted versus $\lambda_{0}$, the photon wavelength in vacuum, along with the argument of $r, \arg (r)$, which is the relative phase shift of the SPP wave upon reflection at the slit grating. The case of a five-slit grating (as in the experiment) is considered, as well as gratings with $N=10, N=20$, and $N=\infty$, in order to highlight the influence of the number of slits on the spectral broadness of the plasmonic lens response. In all cases, the slit period is $P=700 \mathrm{~nm}$. The reflection coefficient $R$ exhibits a sharp peak centered at $\lambda_{0}=700 \mathrm{~nm}$, which becomes narrower and more intense with increasing $N$. For $N=5$, the peak intensity is $R=0.13$ and its width is about $60 \mathrm{~nm}$. The reflection peak coincides with a sharp rise in the transmission coefficient $T$, with a local maximum of $T=$ 0.23 at $\lambda_{0}=700 \mathrm{~nm}$. Since $R+T+S+A=1$ as discussed 

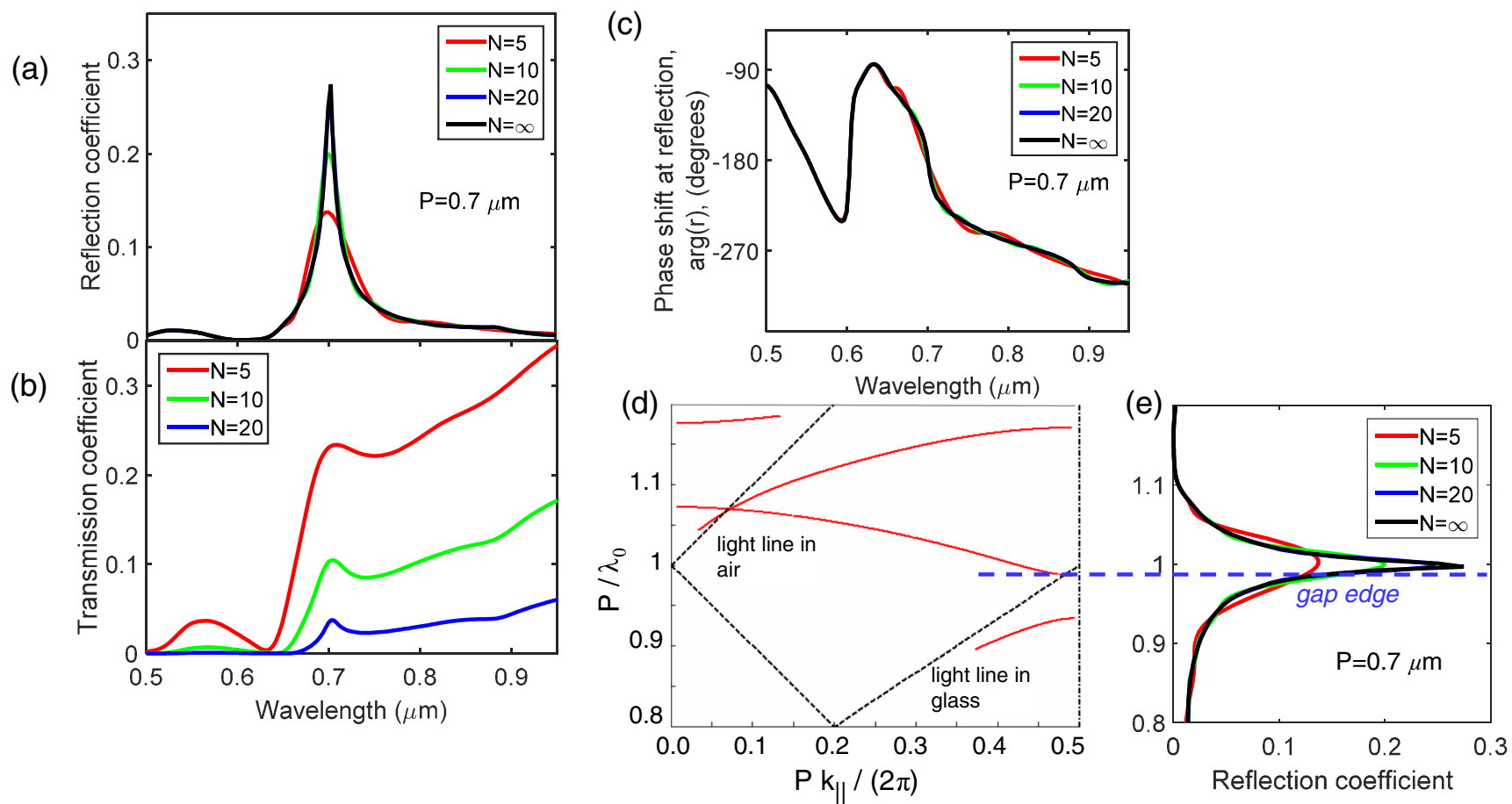

FIG. 6. Spectral response of the slit grating to incident SPP waves: numerical calculations. (a) and (b) Theoretical SPP reflection and transmission coefficients, $R=|r|^{2}$ and $T=|t|^{2}$, and (c) phase shift at reflection, i.e., the argument of the complex amplitude reflection coefficient, $\arg (r)$, of a slit grating with period $P=700 \mathrm{~nm}$. The results are shown for a number of slits $N=5, N=10, N=20$ and [only for $R$ and $\arg (r)] N=\infty$, i.e., an infinite number of slits. (d) Dispersion relation for the slit grating with $P=700 \mathrm{~nm}$. The black dashed lines labeled light lines correspond to the folded dispersion lines of photons propagating in air and glass along directions parallel to the air-glass interface, respectively. (e) SPP reflection coefficient [same data as shown in (a)], plotted with same $Y$ axis as that of the dispersion curves. A horizontal dashed line indicates the energy position of the upper gap edge. Calculation methods are described in Refs. [42] and [43].

above, a concomitant rise in $R$ and $T$ must correspond to a drop in the light emitted in the far field. This has previously been reported for a single linear SPP grating (i.e., for a single isolated grating, which is not part of a cavity) [44], where a peak in $R$ and a dip in $S$ occur when the SPP wavelength matches the grating period.

Recent studies on circular plasmonic structures similar to our plasmonic lens have shown a similar spectral dip; yet, the authors ascribed such a dip to either a Fano resonance [24] due to SPP coupling with a waveguiding layer atop the grating (such a layer is absent in our case), or to an instrumental artifact due to the collection geometry (see the supporting information of Ref. [45]). The latter artifact is specific to the detection scheme used in Ref. [45] (a parabolic mirror with a small hole) and is never present in our case, thanks to the use of an immersion objective lens. Moreover, the spectra shown in Fig. 5 do not systematically exhibit a dip at $\lambda_{0}=P$ or $\lambda_{\text {SPP }}=P$, despite the inference from the results of Fig. 6 that the out-of-plane SPP-to-light scattering coefficient $S$ must be minimal for $\lambda_{0}=P$; in the experiment, a peak or plateau may be seen as well, depending on the inner diameter $D$ of the plasmonic lens. From this, we infer that the cavity modes of the central disk determine whether a peak or a dip appears at a specific energy in the measured spectra, as these modes influence the incident SPP intensity, and are not included in the simulation. Both the modulus and the argument of $r$ determine the magnitude of these features. In Fig. 6(c), we see that the phase $\arg (r)$ varies rapidly at the wavelength values where $r$ is maximum. This is equivalent to a rapidly changing cavity size, which may explain the abrupt changes in the spectrum that are observed around $\lambda_{0}=P$. At this point, we emphasize that the experimentally observed spectra are highly reproducible and that they are not subject to spectral shifts due to STM tip shape modifications [46,47], since the tip material (tungsten) is a nonplasmonic metal in this energy range.

In Figs. 6(d) and 6(e), we explain the optical behavior of the slit grating in terms of the SPP dispersion relation. Here, we use reduced energy and wave-vector variables, $P / \lambda_{0}$ and $P k_{\|} / 2 \pi$, respectively ( $P$ is the grating period and $k_{\|}$is the wave vector in the plane of the interface). The grating periodicity leads to the opening of an energy gap in the dispersion curve. Here the gap exists from $0.94-0.99$ in reduced energy units at a reduced wave vector of 0.5. As is visible in Fig. 6(e), the reflection peak (occurring at energy $P / \lambda_{0}=1$ ) is very close in energy to the upper-energy gap edge in the dispersion relation, confirming previous interpretations reported for linear SPP gratings (i.e., that a maximum in the density of states occurs at the upper edge of the energy gap) $[44,48,49]$. This explains why the strongest intensity variations in the spectra shown in Fig. 5 occur around photon wavelength $\lambda_{0}=P$.

In Fig. 6 we also see that the larger the slit number, the higher and the spectrally narrower the peak in the SPP reflectivity of the slit grating. Increasing $N$ is thus expected to yield stronger and sharper features (peaks and dips) in the spectral response of the plasmonic lens, which may be considered a drawback, depending on the targeted applications. Thus, the 
following design rule for light beam microsources based on circular slit gratings may be enunciated: by limiting the slit number, a smaller spatial footprint and a broader spectral response of the plasmonic structure are obtained at the expense of emission directivity. A broad spectral response may indeed be crucial if, e.g., such a microsource is used for optical transmission with wavelength multiplexing.

\section{CONCLUSION}

In conclusion, in this paper we introduce an experimental method combining STM, optical microscopy, and optical spectroscopy for carrying out angle-resolved optical spectroscopy measurements. This technique is used to analyze the light emitted from a plasmonic structure upon local, low-energy electrical excitation with tunnel electrons. Thus, we characterize the emission pattern and spectral response of a plasmonic lens consisting of concentric circular slits etched in a thick gold film. Such a design is very attractive for building an electrically driven microsource of highly collimated light since its spectral response is broad and optimal within a wide energy range, especially in comparison to other resonant plasmonic structures such as Yagi-Uda optical antennas [50]. In this way, a very simple broadband electrical SPP source, such as a tunnel nanojunction, can drive such light beam microsources. We demonstrate that such a periodic structure with a limited number of slits, i.e., $N=5$, offers a fair tradeoff between low angular beam divergence, small spatial footprint, and broadband spectral response.

\section{ACKNOWLEDGMENTS}

The financial support of the China Scholarship Council (CSC) (No. 201304910386) is gratefully acknowledged. The authors thank J.-F. Motte and G. Julie at the NanoFab facility, Institut Néel in Grenoble for the fabrication of the plasmonic structures.
[1] Y. Fu and X. Zhou, Plasmonics 5, 287 (2010).

[2] J. M. Steele, Z. Liu, Y. Wang, and X. Zhang, Opt. Express 14, 5664 (2006).

[3] W. Chen, D. C. Abeysinghe, R. L. Nelson, and Q. Zhan, Nano Lett. 9, 4320 (2009).

[4] A. Yanai and U. Levy, Opt. Express 17, 924 (2009).

[5] P. Wróbel, J. Pniewski, T. J. Antosiewicz, and T. Szoplik, Phys. Rev. Lett. 102, 183902 (2009).

[6] S. Yue, Z. Li, J. Chen, and Q. Gong, Appl. Phys. Lett. 98, 161108 (2011).

[7] J. Ji, Y. Meng, and J. Zhang, J. Nanophoton. 9, 093794 (2015).

[8] Y. C. Jun, K. C. Huang, and M. L. Brongersma, Nat. Commun. 2, 283 (2011).

[9] M. G. Harats, N. Livneh, G. Zaiats, S. Yochelis, Y. Paltiel, E. Lifshitz, and R. Rapaport, Nano Lett. 14, 5766 (2014).

[10] S. Cao, E. Le Moal, E. Boer-Duchemin, G. Dujardin, A. Drezet, and S. Huant, Appl. Phys. Lett. 105, 111103 (2014).

[11] Q. Zhan, Adv. Opt. Photon. 1, 1 (2009).

[12] Y. Uehara, Y. Kimura, S. Ushioda, and K. Takeuchi, Jpn. J. App. Phys. 31, 2465 (1992).

[13] T. Wang, E. Boer-Duchemin, Y. Zhang, G. Comtet, and G. Dujardin, Nanotechnol. 22, 175201 (2011).

[14] P. Bharadwaj, A. Bouhelier, and L. Novotny, Phys. Rev. Lett. 106, 226802 (2011).

[15] Y. Zhang, E. Boer-Duchemin, T. Wang, B. Rogez, G. Comtet, E. Le Moal, G. Dujardin, A. Hohenau, C. Gruber, and J. R. Krenn, Opt. Express 21, 13938 (2013).

[16] J. T. van Wijngaarden, E. Verhagen, A. Polman, C. E. Ross, H. J. Lezec, and H. A. Atwater, Appl. Phys. Lett. 88, 22111 (2006).

[17] F. J. G. de Abajo, Rev. Mod. Phys. 82, 209 (2010).

[18] D. T. Schoen, T. Coenen, F. J. García de Abajo, M. L. Brongersma, and A. Polman, Nano Lett. 13, 188 (2013).

[19] D. Hone, B. Mühlschlegel, and D. J. Scalapino, Appl. Phys. Lett. 33, 203 (1978).

[20] N. Yu, R. Blanchard, J. Fan, Q. J. Wang, C. Pflügl, L. Diehl, T. Edamura, M. Yamanishi, H. Kan, and F. Capasso, Opt. Express 16, 19447 (2008).
[21] C. E. Hofmann, E. J. R. Vesseur, L. A. Sweatlock, H. J. Lezec, F. J. G. de Abajo, A. Polman, and H. A. Atwater, Nano Lett. 7, 3612 (2007).

[22] H. Aouani, O. Mahboub, E. Devaux, H. Rigneault, T. W. Ebbesen, and J. Wenger, Nano Lett. 11, 2400 (2011).

[23] P. Ginzburg, A. Nevet, N. Berkovitch, A. Normatov, G. M. Lerman, A. Yanai, U. Levy, and M. Orenstein, Nano Lett. 11, 220 (2011).

[24] N. Livneh, M. G. Harats, S. Yochelis, Y. Paltiel, and R. Rapaport, ACS Photon. 2, 1669 (2015).

[25] P. Rai, N. Hartmann, J. Berthelot, J. Arocas, G. C. des Francs, A. Hartschuh, and A. Bouhelier, Phys. Rev. Lett. 111, 026804 (2013).

[26] J. Kern, R. Kullock, J. Prangsma, M. Emmerling, M. Kamp, and B. Hecht, Nat. Photon. 9, 582 (2015).

[27] M. Parzefall, P. Bharadwaj, A. Jain, T. Taniguchi, K. Watanabe, and L. Novotny, Nat. Nanotechnol. 10, 1058 (2015).

[28] N. Cazier, M. Buret, A. V. Uskov, L. Markey, J. Arocas, G. C. Des Francs, and A. Bouhelier, Opt. Express 24, 3873 (2016).

[29] M. Aeschlimann, M. Bauer, D. Bayer, T. Brixner, S. Cunovic, A. Fischer, P. Melchior, W. Pfeiffer, M. Rohmer, C. Schneider, C. Strüber, P. Tuchscherer, and D. V. Voronine, New J. Phys. 14, 033030 (2012).

[30] C. Lemke, T. Leißner, A. Evlyukhin, J. W. Radke, A. Klick, J. Fiutowski, J. Kjelstrup-Hansen, H.-G. Rubahn, B. N. Chichkov, C. Reinhardt, and M. Bauer, Nano Lett. 14, 2431 (2014).

[31] B. Rogez, S. Cao, G. Dujardin, G. Comtet, E. Le Moal, A. Mayne, and E. Boer-Duchemin, Nanotechnol. 27, 465201 (2016).

[32] T. Wang, G. Comtet, E. Le Moal, G. Dujardin, A. Drezet, S. Huant, and E. Boer-Duchemin, Opt. Lett. 39, 6679 (2014).

[33] B. Rogez, R. Horeis, E. Le Moal, J. Christoffers, K. Al-Shamery, G. Dujardin, and E. Boer-Duchemin, J. Phys. Chem. C 119 , 22217 (2015).

[34] T. Coenen, E. J. R. Vesseur, and A. Polman, Appl. Phys. Lett. 99, 143103 (2011). 
[35] T. Coenen, E. J. R. Vesseur, A. Polman, and A. F. Koenderink, Nano Lett. 11, 3779 (2011).

[36] See Supplemental Material at http://link.aps.org/supplemental/ 10.1103/PhysRevB.96.115419 for experimental spectra of the light emitted upon STM excitation of single circular slits and generated data confirming the role of SPP cavity modes, and the procedure for correcting experimental spectra for the detection efficiency of our setup.

[37] P. Johansson, R. Monreal, and P. Apell, Phys. Rev. B 42, 9210 (1990).

[38] P. Johansson, Phys. Rev. B 58, 10823 (1998).

[39] T. Wang, E. Boer-Duchemin, G. Comtet, E. Le Moal, G. Dujardin, A. Drezet, and S. Huant, Nanotechnol. 25, 125202 (2014).

[40] K. Joulain, R. Carminati, J.-P. Mulet, and J.-J. Greffet, Phys. Rev. B 68, 245405 (2003).

[41] L. Douillard and F. Charra, J. Phys. D: Appl. Phys. 44, 464002 (2011).
[42] Q. Cao, P. Lalanne, and J.-P. Hugonin, J. Opt. Soc. Am. A 19, 335 (2002).

[43] J. P. Hugonin and P. Lalanne, J. Opt. Soc. Am. A 22, 1844 (2005).

[44] F. López-Tejeira, F. García-Vidal, and L. Martín-Moreno, Appl. Phys. A 89, 251 (2007).

[45] C. I. Osorio, T. Coenen, B. J. M. Brenny, A. Polman, and A. F. Koenderink, ACS Photon. 3, 147 (2016).

[46] J. Aizpurua, S. P. Apell, and R. Berndt, Phys. Rev. B 62, 2065 (2000).

[47] E. Le Moal, S. Marguet, D. Canneson, B. Rogez, E. BoerDuchemin, G. Dujardin, T. V. Teperik, D.-C. Marinica, and A. G. Borisov, Phys. Rev. B 93, 035418 (2016).

[48] F. Pincemin and J.-J. Greffet, J. Opt. Soc. Am. B 13, 1499 (1996).

[49] T. Søndergaard, S. I. Bozhevolnyi, and A. Boltasseva, Phys. Rev. B 73, 045320 (2006).

[50] H. F. Hofmann, T. Kosako, and Y. Kadoya, New J. Phys. 9, 217 (2007). 